\title{
Platelets: an outlook from biology through evidence-based achievements in critical care
}

\author{
Rubens C. Costa-Filho', ${ }^{1,2}$ Fernando A. Bozza ${ }^{3,4}$ \\ ${ }^{1}$ Department of Critical Care, Hospital Pro Cardíaco, Rua Gal. Polidoro, Rio de Janeiro RJ, Brazil; ${ }^{2}$ Trombocore, Haemostasis \& Thrombosis \\ Studies with roTEM ${ }^{\circledR}$ thromboelastometry directed to critically ill patients, Rua Dona Mariana, Botafogo, Rio de Janeiro RJ, Brazil; ${ }^{3}$ Instituto D’Or \\ de Pesquisa e Ensino (IDOR), Rua Diniz Cordeiro, Rio de Janeiro RJ, Brazil; ${ }^{4}$ Instituto Nacional de Infectologia Evandro Chagas, FIOCRUZ, Estr. \\ de Manguinhos, Manguinhos, Rio de Janeiro RJ, Brazil \\ Contributions: (I) Conception and design: RC Costa-Filho; (II) Administrative support: None; (III) Provision of study materials or patients: FA Bozza; \\ (IV) Collection and assembly of data: RC Costa-Filho; (V) Data analysis and interpretation: RC Costa-Filho; (VI) Manuscript writing: All authors; \\ (VII) Final approval of manuscript: All authors. \\ Correspondence to: Rubens C. Costa-Filho. Praça Atahualpa 60 part 310, Leblon, Rio de Janeiro, CEP 22441-130, Brazil. Email: rubenscosta@me.com.
}

\begin{abstract}
Since the original observations by Bizzozero and Osler, we have seen tremendous advances in the understanding of platelets far beyond haemostasis and the restoration of injured endothelium. In this minireview on platelets, we will briefly outline their historical description and the importance of their evolution, focusing on a 450 million years old living fossil of Limulus polyphemus, a marine chelicerate arthropod, which helped researchers explain the basis for the immunity role of platelets and make correlations with platelet ultrastructure and function. In addition, the impact of the Limulus Amoebocyte Lysate (LAL) test for modern medicine is highlighted. The role of platelets in cardiovascular diseases, their relevance in arterial and venous thrombosis, and the utilization of antithrombotic drugs as therapeutic agents are also reported. Furthermore, platelet receptors are crucial in aggravating or mitigating other diseases, such as cancer and infections, which can recruit cells and have numerous interactions in a process recently coined "NETosis formation", which is also briefly depicted.
\end{abstract}

Keywords: Platelet; thrombosis; antithrombotic drugs; Limulus; Limulus Amoebocyte Lysate (LAL) test; cancer; protease-activated receptor 1 (PAR-1); glycoprotein (GP) Ib/IX/V complex; purinoceptor; NETosis

Submitted Aug 13, 2017. Accepted for publication Nov 01, 2017.

doi: 10.21037/atm.2017.11.04

View this article at: http://dx.doi.org/10.21037/atm.2017.11.04

\section{A brief history of platelet descriptions}

The first description of platelets in blood is dated at the end of the $18^{\text {th }}$ century. However, it was only between 1865 and 1877 that these "discoid corpuscles" were clearly depicted, although without a complete understanding their origin, significance or function. For example, in 1874, Osler was not sure if platelets were normal blood elements because of their disk-like structure that circulated freely in the blood, but when removed from vessels, formed aggregates $(1,2)$. At that time, Osler and others had reported that these corpuscular structures were possibly degenerated and disintegrated leukocytes or clots of fibrin or a particular kind of microbe $(1,3)$.

However, it was in 1881 that the Italian pathologist Giulio Bizzozero (1846-1901), based on his remarkable haematological investigation (Figure 1), for the first time identified platelets as a third morphological element of the blood, unrelated to erythrocytes or leukocytes. Bizzozero described "discoid corpuscles" without a nucleus, consisting of a membrane and a matrix in which there were a few dispersed granules. He called these "discoid corpuscles" blood piastrine (the Italian for small plates; they were called Blutplättchen in German, and Petites plaques in Frenchsubsequently called plaquettes, and in English, they were later named "platelets"). He observed them in vivo 

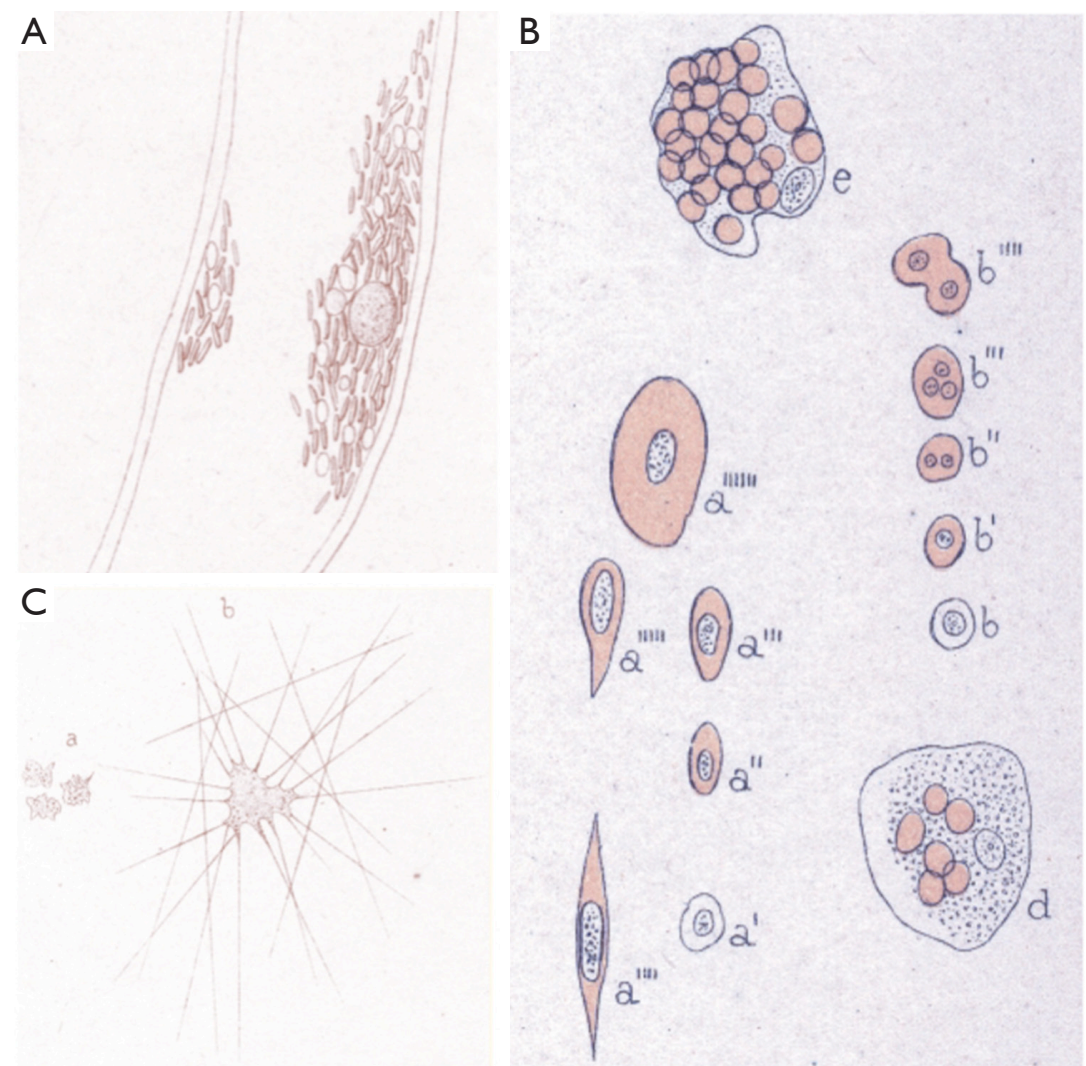

Figure 1 Bizzozero drawings from reference (4). (A) Parietal thrombi among platelets is a small white blood cell; (B) a-platelets observed immediately after blood extraction; b-platelets after eight minutes, when they are found fused into a single mass with fibrin threads merging within the aggregates; (C) Bizzozero's drawings showing developmental stages of red blood cells. Series shows red blood cells in the bone marrow of a frog, series b shows red blood cells in the bone marrow of humans, and d and e show macrophages that have phagocytized erythrocytes (4).

under the microscope as colourless, transparent particles, approximately $2-3 \mu \mathrm{m}$ in diameter (4). Additionally, his utmost achievement was establishing the central role of platelets, not only in physiological haemostasis but also in thrombosis, in an era when knowledge about the mechanisms of blood coagulation was still largely incomplete.

\section{Evolution of mammalian platelets}

Mammalian platelets originate from the cytoplasm of megakaryocytes (MKs), a unique polyploid haematopoietic cell. In all other animal species, cells involved in haemostasis and blood coagulation are nucleated and named thrombocytes, to distinguish them from non-nucleated platelets (5). The amoebocyte is the only cell of the Limulus polyphemus (Horseshoe crab) designated to transport oxygen and react to non-self elements, and it acts similarly to human platelets with regard to coagulation. This unique cell provides the basis for the understanding of the many non-haemostatic functions of platelets $(5,6)$. The activation of the Limulus amoebocyte triggers the initiation of coagulation but is also associated with the induction of other defence mechanisms, such as wound healing, the activation of the complement system, and the release of antibacterial factors. The functions performed by amoebocytes resemble the mammalian processes that involve leukocytes, platelets and other cells. Even after more than 450 million years of evolution, mammalian platelets have preserved many of the functions of Limulus amoebocytes. The understanding of their functions (platelet and amoebocyte) has changed in parallel with new discoveries. However, coagulation and antibacterial mechanisms remain at least partially linked. An example is the relationship between the haemostatic 
and antibacterial activities in platelets when Staphylococcus aureus induces platelet aggregation through a fibrinogendependent mechanism (7).

\section{Limulus polyphemus (horseshoe crabs) and the Limulus Amoebocyte Lysate (LAL) test}

Four species of horseshoe crabs exist today. The Atlantic horseshoe crabs are most commonly found on shallow ocean grounds in the Gulf of Mexico and along the northeastern coast of the US. The other three species are found in Southeast Asia. Horseshoe crabs are not true crabs, neither crustacean; they are marine arthropods more closely related to arachnids (a group that includes spiders and scorpions) than to crustaceans (a group that includes true crabs, lobsters, and shrimp). Horseshoe crabs are often called "living fossils" because their ancestors date back to 450 million years before the Triassic period (8). Horseshoe crabs have been an object of zoological research for almost 200 years (9). In the quality assurance of medical products, tests for sterility are essential. For parenteral pharmaceuticals (vaccines, infusion drugs, and medical devices), avoiding the presence of pyrogens is crucial. Additionally, enzymes from their blood have been used by astronauts on the International Space Station to test surfaces for unwanted microorganisms (10). The LAL test assay was incorporated as one more official method used by NASA's planetary protection program to certify the purity of interplanetary robotic spacecraft prior to departure (https://planetaryprotection.jpl.nasa.gov/resources/img/ content/planetary-implementation-pub.pdf).

Many fever-inducing substances (endotoxins and non-endotoxins) cannot be eliminated with standard sterilisation processes and are biologically active once in the bloodstream, causing risks to human health, ranging from mild reactions (e.g., fever) to septic shock and death. Over the years, various pyrogen testing methods have been introduced, namely: in the 1940s, the rabbit pyrogen test, which is an in vivo test that measures the fever reaction as an endpoint; in the 1970s, the LAL test, which is an in vitro test (with the haemolymph of the horseshoe crab) that specifically detects endotoxin; and in 2010, the MonocyteActivation Test (MAT), which is a non-animal based in vitro pyrogen test that represents a full replacement of the rabbit test (11). Limulus is enormously important to the biomedical industry because its copper blue-based blood contains coagulant proteins called LALs, which are very similar with the platelets granules and coagulate in the presence of minimum amounts of bacterial toxins. The horseshoe crab's blood has the mysterious ability to coagulate bacterial contamination (higher sensitivity for non-self cells) at as little as one part per trillion. The FDA requires the use of LAL to test all injectable and intravenous drugs and equipment that require evaluation for contamination and are produced in the US. No other test works so perfectly or so quickly to detect contamination. Every year, the medical testing industry catches approximately half a million of these arthropods to collect approximately a third of their blood into a blood bank station before releasing them alive to the sea (blood extraction is shown in Figure 2). Notably, there is cumulative concern among scientists that biomedical entities may be placing Limulus in danger. In addition, The International Union for Conservation of Nature has already labelled the American Horseshoe crab "vulnerable" to extinction in 2016. Importantly, global pharmaceutical markets are expected to grow as much as $8 \%$ over the next year, while America's medical devices market is expected to grow by $25 \%$ by 2020 (12). Thus, the LAL test is of crucial importance to medicine (Figure 2).

\section{Development and platelet formation}

As a non-Newtonian fluid, blood makes platelets circulate near the outside edge of the bloodstream but is influenced by their viscosity, mainly as assessed by haematocrit. At the apical surface of the endothelium, platelets are easily detected when vascular damage has occurred. This phenomenon validates a remarkable increase in our understanding regarding the roles of platelets in haemostasis, thrombosis and wound repair (13). Correspondingly, current evidence has shown that platelets are entangled in many other physiological and pathological processes beyond haemostasis and thrombosis such immuno-thrombosis, the development of atherosclerosis, lymphatic vessel enlargement, angiogenesis and tumour metastasis (14-16).

Platelets usually circulate at a concentration of $150 \times 10^{9}-$ $400 \times 10^{9}$ platelets $/ \mathrm{L}$ in the blood, which means that 100 billion platelets are produced and released per day from MKs in the bone marrow. MKs originate from pluripotent stem cells and undergo multiple DNA replications without cell division by a single process called endomitosis. Platelet-specific organelles, proteins, membrane systems, and others contents essential for platelet function are formed after megakaryocyte maturation. The latest evidence demonstrates that MKs release platelets within 

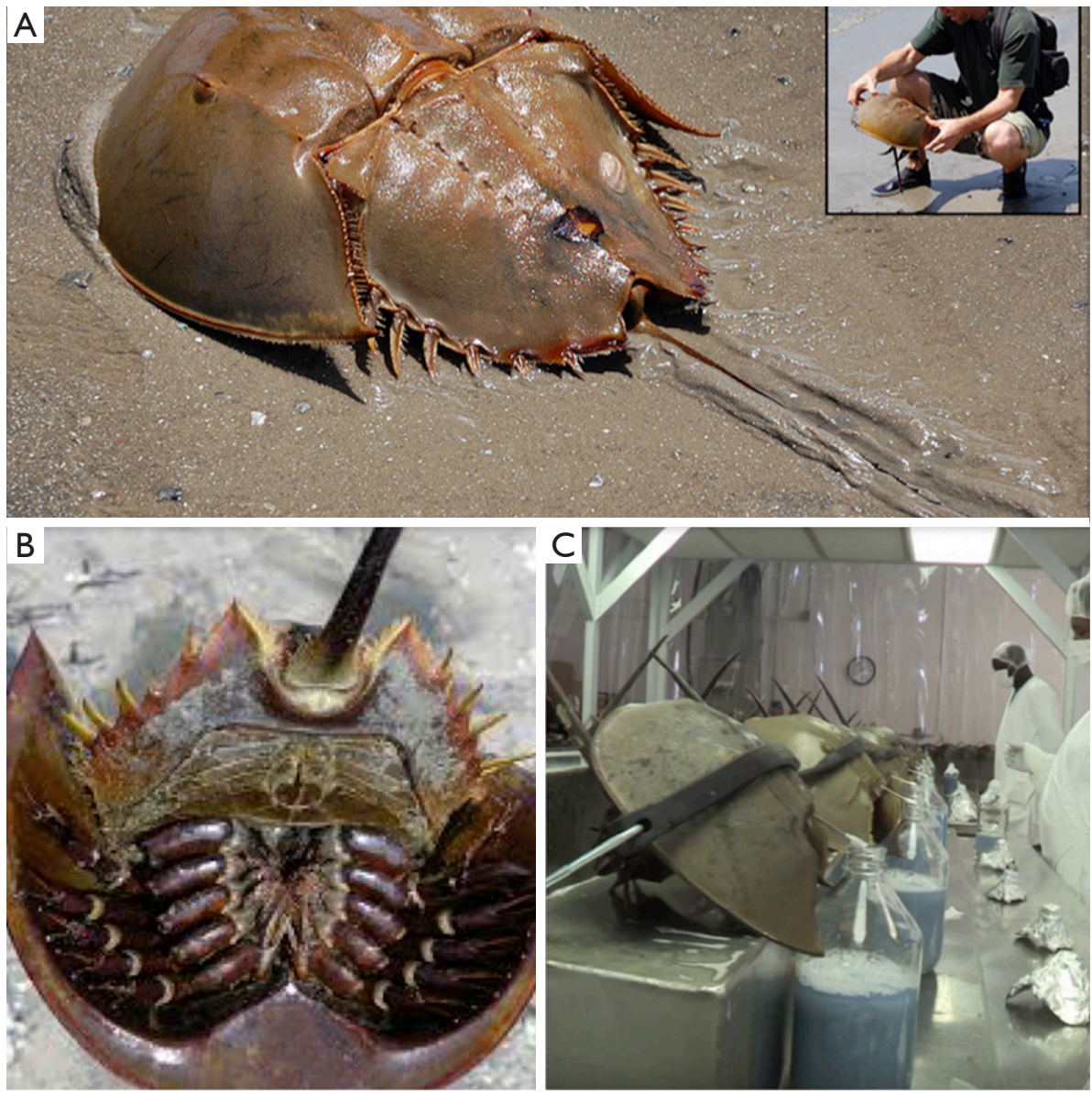

Figure 2 Some characteristics of horseshoe crab (Limulus polyphemus). (A) L. polyphemus can be up to $60 \mathrm{~cm}$ (24 in) long (including the tail) (12); (B) the illustration shows the underside of a specimen of chelicerae; (C) blood extraction from L. polyphemus in Delaware to produce LAL test. They are returned to the sea alive.

the pulmonary vasculature (17) in addition to the bone marrow. Aschoff was the first to describe pulmonary MKs in 1893 (18) and suggested that they came from the bone marrow, then migrated into the bloodstream, and because of their massive size, were lodged in the capillary beds of the lung, where they released platelets (19).

Many different signal transduction pathways, such as JAK, STAT, and MAPK, are successively activated and help the survival, proliferation and differentiation of bone marrow MKs with concomitant augmentation of platelet production. Intriguingly, recent evidence shows a new mechanism by which MKs can generate platelets via IL-1 $\alpha(20)$.

Human platelets have a short-lived existence of up to 10 days. Phagocytic cells eliminate the majority of platelets in the reticuloendothelial system (RES), such as macrophages in the spleen. Numerous mechanisms can mediate platelet clearance, including antibody-independent and antibody-dependent pathways. Antibody-dependent platelet elimination has both antibody Fc-dependent and Fc-independent mechanisms. Remarkably, platelets contain an intrinsic apoptotic programme that can regulate their survival; precisely, stimulation of pro-death proteins Bak/ Bax facilitates platelet mitochondrial outer membrane permeability. However, the triggering of apoptosis can prime P-selectin exposure, surface receptor shedding, loss of mitochondrial membrane potential, caspase activation, and microparticle generation with phosphatidylserine (PS) expression (21-23) - these characteristics share features of platelet storage lesions (PSL) or platelet storage defects (PSD). Thus, apoptotic platelets become procoagulant due to PS exposure, which is also a hallmark of cell death, 
acting as an "eat me" signal that promotes engulfment by phagocytes (19).

\section{Platelet receptors as therapeutic targets}

Activated platelets deliver negatively charged surfaces that harbour important steps for coagulation factors, forming Prothrombinase (Xa-Va) and Tenase (IXa-VIIIa) complexes in order to generate huge amounts of thrombin $\left(1 \times 10^{6}\right)$ according to the cell-based thrombin generation model described by Hoffman et al. in 2001 (24,25). The recently described concept of a "protein wave of haemostasis" may indicate a potential role of platelet-released plasma fibronectin in thrombosis and haemostasis mechanisms (26). Moreover, platelets are key mediators of atherothrombosis, which are actively involved in all three waves of thrombus formation (27): (I) protein wave; (II) platelet accretion; and (III) blood coagulation.

Platelet receptor adhesion molecules, including IIb3 integrin and glycoprotein (GP) Ib/IX/V complex, are essential for these processes. Many others are involved, such as P-selectin, GPVI PAR-1 (proteinase-activated receptor 1) and $\mathrm{P} 2 \mathrm{Y}$ purinoceptor $1\left(\mathrm{P}_{2} \mathrm{Y}_{1}\right)$ and $\mathrm{P}_{2} \mathrm{Y}_{12}$ (28) (Figure 3). Still, there are three important groups of important platelet receptors that require positive feedback loops for their activation:

(I) $\mathrm{P} 2 \mathrm{Y}_{1}$ and $\mathrm{P} 2 \mathrm{Y}_{12}$ are stimulated by adenosine diphosphate (ADP) released from platelet-dense granules $(\delta-G)$;

(II) 2-5-hydroxytryptamine $2 \mathrm{~A}$ receptors $\left(5 \mathrm{HT}_{2 \mathrm{~A}}\right)$, which are stimulated by $5-\mathrm{HT}$ or serotonin released from platelet- $\delta-G$ (not shown in Figure 3);

(III) The thromboxane prostanoid (TP) receptor is stimulated by thromboxane A2 (TxA2), which is generated by the platelet cyclooxygenase 1 (COX1)-dependent signalling pathway (Figure 3).

Otherwise, platelet-to-platelet aggregation is mediated by fibrinogen (GPIIb/IIIa) and, at high shear flow, by von Willebrand factor (vWF) binding (GPIb) to the activated molecular conformation of integrin $\alpha \operatorname{IIb} \beta 3$, which is the receptor with the highest copy number $(\sim 80,000$ per platelet) (30). Additionally, there are interactions with other cells, such as adhesion between platelet-monocytes, that are initially mediated by the binding of platelet surface $\mathrm{P}$-selectin (expressed on the platelet surface after platelet degranulation) to its constitutively expressed cognate receptor, P-selectin glycoprotein ligand 1 (PSGL1), on the monocyte surface (30).
All receptors play important roles in normal haemostasis and in disease. This can be demonstrated in some congenital bleeding disorders such as Glanzmann thrombasthenia (platelets contain defective or low levels of GPIIb/IIIa) and Bernard-Soulier syndrome (also called haemorrhagiparous thrombocytic dystrophy, a bleeding disorder caused by a deficiency in GPIb) (30), as well as in acquired disorders when bleeding complications emerge secondary to the administration of receptor antagonists. In addition, under pathological conditions, excessive platelet activation along with endothelial dysfunction is a perfect milieu to cause thrombotic diseases, such as myocardial infarction and ischaemic stroke, which cause far more deaths each year than cancer or respiratory diseases (31). However, many patients undergoing therapies with antiplatelet agents (even combined dual antiplatelet therapy), still suffer ischaemic events since a state called "platelet hyperactivity" may be present, such as in patients with diabetes, which may contribute along with others factors including drug interactions and genetic profiles. The contemporary knowledge in this field has been observed in many clinical trials (32). There are several worthwhile available reviews regarding these trials, evaluating ADP antagonists (e.g., $\mathrm{P} 2 \mathrm{Y}_{12}$ inhibitors), thromboxane antagonists and PAR-1/4 inhibitors (32-34). Figure 3 highlights the therapeutic stages of development of some drugs that target platelet adhesion molecules and the recent advances in drug development.

\section{Antiplatelet therapies applied in arterial thrombosis}

Thrombosis can be caused by plaque rupture, exposition of subendothelial matrix proteins, such as collagen, vWF, fibrinogen, fibronectin and laminin, leading to a rapid platelet response (28). Inappropriate platelet adhesion, activation and aggregation promotes excessive platelet plug formation, which causes vascular obstruction and severe disease. Current FDA-approved antiplatelet therapies are shown in Figure 3. Such therapies are aimed at the following: inhibiting TXA2 synthesis, which downregulates excess activation (e.g., aspirin and triflusal); antagonizing the function of platelet $\mathrm{P} 2 \mathrm{Y}$ receptors, which are activated by $\mathrm{ADP}$, (e.g., clopidogrel, prasugrel and ticagrelor); inhibiting platelet integrin IIb3 activity, which restrains platelets aggregation (e.g., abciximab, eptifibatide and tirofiban); inhibiting phosphodiesterase, which increases platelet internal cAMP/cGMP levels (e.g., dipyridamole and cilostazol); and inhibiting PAR-1 receptors, which 


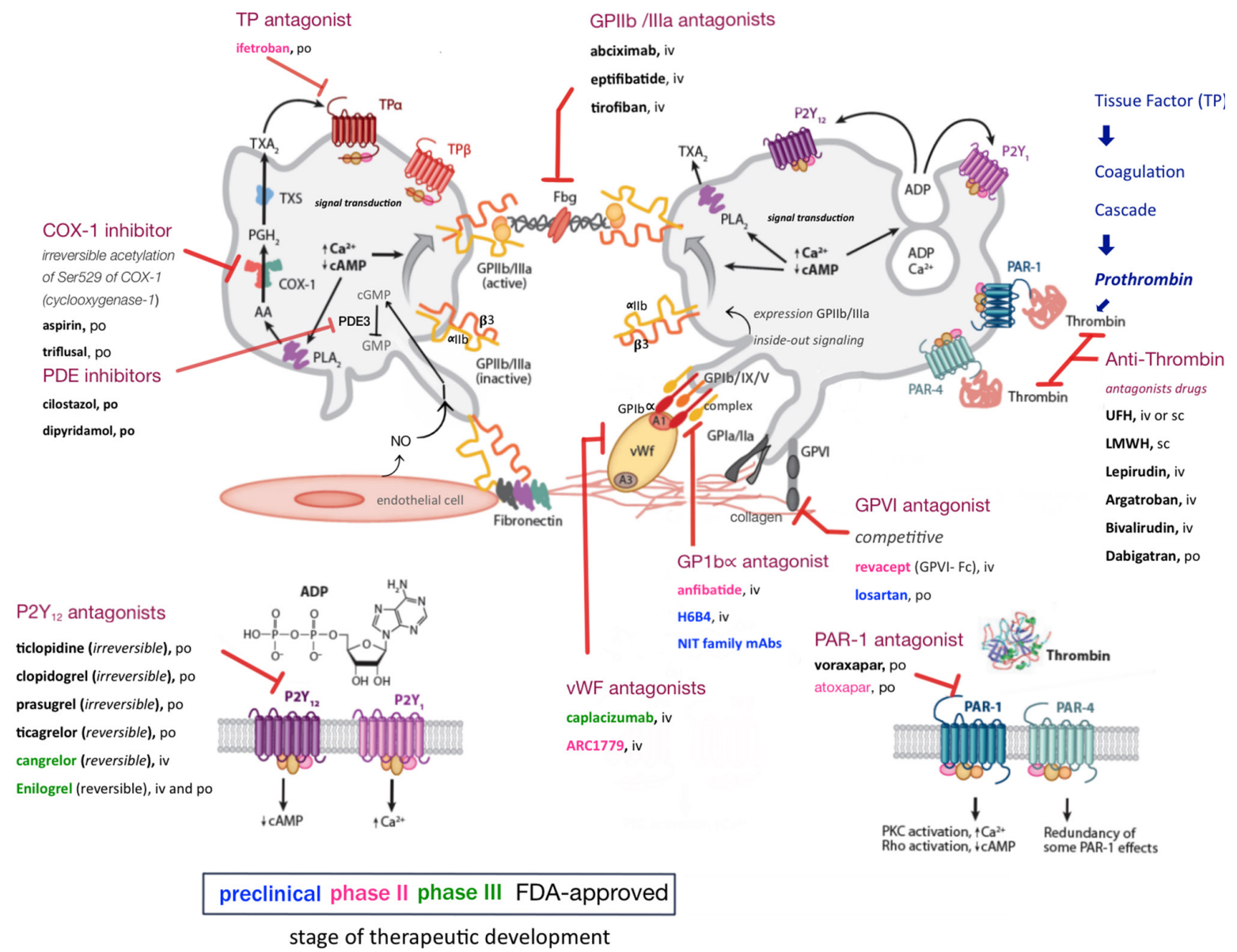

Figure 3 Schematic diagram of platelet receptors and sites of drugs actions. Platelets undergo activation at the sites of injury, with subsequent release of soluble components, including ADP from alfa-granules. Through autocrine and paracrine stimulation, ADP binds to the P2Y12 and P2Y1 receptors, which start and amplify platelet activation and aggregation. In addition, TxA2 is produced by activated platelets, further contributing to sustained activation and aggregation via the TP, which enhances signal transduction. Thrombin also activates two surface platelet receptors: PAR-1 and 4 (PAR-1 and PAR-4) which leads to a rapid platelet shape change and granule release. $\mathrm{NO}$ is released by intact endothelium, which has antithrombotic and vasodilatory effects. By binding sGC, it rapidly upregulates the intracellular cGMP and cAMP. The increase in cGMP levels is accompanied by a decrease in intracellular Ca2+ flux and inhibits the conformational change of GPIIb-IIIa into an active form, thus decreasing platelet association with fibrinogen. Elevated levels of cGMP diminish alpha granule secretion. PDE inhibitors promote sustained levels of cGMP and cAMP, which influence signal transduction for platelet activation (29). Targeting various pathways (ADP, TxA2, thrombin) with different drugs have been developed and tested in recent decades. Many types of mobile transmembrane receptors are present at the PLT membrane, including (not displayed in Figure 3) many integrins ( $\alpha \operatorname{IIb} \beta 3, \alpha 2 \beta 1, \alpha 5 \beta 1, \alpha 6 \beta 1, \alpha \mathrm{V} \beta 3$ ), LRR receptors (displayed in Figure 3) (GP Ib/IX/V, toll-like receptors), GPCR, PAR-1 and PAR-4, thrombin receptors, $\mathrm{P} 2 \mathrm{Y} 1$ and $\mathrm{P} 2 \mathrm{Y} 12$ ADP receptors, TP $\alpha$ and TP $\beta$ (TxA2 receptors), proteins belonging to the immunoglobulin superfamily (GP VI, Fc $\gamma$ RIIA), C-type lectin receptors (P-selectin) (not displayed), tyrosine kinase receptors (thrombopoietin receptor, Gas6, ephrins, and Eph kinases) (not displayed), and a miscellaneous number of other receptors (CD63, CD36, PSGL1, TNF receptor type, etc.) (not displayed). The plasma membrane is the site where a multitude of receptors are present that can interact with soluble ligands, with cellular counter receptors on other PLTs, on leucocytes, or on endothelial cells, with molecules of the extracellular matrix but also with pathogens. These receptors play an important role in inside-outside signalling in the course of PLT activation and the release reaction. This template provides an overview of the available agents as well as of their main targets and their respective pathways and stages of development towards activation. ADP, adenosine diphosphate; TP, thromboxane prostanoid receptor; NO, nitric oxide; sGC, soluble guanylyl cyclase; cGMP, cyclic guanosine 5'-monophosphate; cAMP, cyclic adenosine monophosphate; PLT, platelets; LRR, leucine-rich repeat; GP, glycoprotein; GPCR, G-protein coupled seven-transmembrane receptors. 
blocks activation caused by thrombin (e.g., voraxapar) (35). All these drugs have helped to prevent cardiovascular deaths, but it is important to mention that weak inhibition of platelet function, excessive bleeding, thrombocytopenia and unexpected platelet activation may occur but are side effects that continuously drive therapeutic research and enhancements in healthcare. Approximately 2,200 Americans die of cardiovascular diseases each year, which is an average of 1 death every 40 second (36). In 2014, the FDA approved voraxapar, a novel antagonist of the thrombin receptor PAR-1, leading to a reduced risk of heart attack and stroke in arteriosclerosis or peripheral arterial disease. This new drug must be avoided in patients with stroke, transient ischaemic attack (TIA) or intracranial haemorrhage since it may increase the risk of intracranial bleeding (37-39). Consequently, therapies aimed at key pathways of platelet activation, including TxA2 synthesis, ADP-mediated signalling and integrin $\alpha I I b \beta 3$ (GPIIb-IIIa) signalling, and other GP antagonists, all have been recognized as mainstays in the management of cardiovascular arterial disease.

\section{Platelet features beyond haemostasis}

Outside their fundamental role in haemostasis and thrombosis, platelets additionally have an important role in wound healing, inflammation, antimicrobial host defence, angiogenesis, tumour growth and metastasis (40-44) (Figure 4). Essentially, mammalian platelet anatomy comprises granules (dense, lysosomal and $\alpha$-granules), mitochondria (Mit), a cytoskeleton, a surface-connected canalicular system and a dense tubular system (DTS), but no nucleus (46), unlike all other animal species, where cells involved in haemostasis are nucleated (shown in Figure 5). Once platelets encounter surface-bound activating factors, such as vWF that are bound to collagen, they induce soluble factors, are contained in granules, and are released into the blood. Subsequently, they avidly react to, bind, spread, secrete and interact with one another, and with fibrin, to form a plug that seals the damaged endothelium. When activated, the platelet membrane invaginates to form a tubular network, the canalicular system, which provides a channel for the release of the granule contents $(46,47)$. Additionally, platelets can mediate leukocyte recruitment not only in cardiovascular disease but also in multiple inflammatory conditions. Elevated levels of circulating platelet-leukocyte aggregates are found in patients suffering from various thrombotic or inflammatory conditions and serve as a surrogate marker for platelet activation. Today these are well-described by the NETosis processes represented in Figure 6. The pathophysiological consequences of these interactions in various diseases such as liver, renal, and skin disease, rheumatoid arthritis, lupus, transplantation, respiratory disorders, viral and bacterial infections, and cancer are discussed by Schrottmaier et al. in a remarkable review (49). Furthermore, platelets have been involved when the basal barrier of the alveolar capillary endothelium loses its integrity in order to repair and remodel in pulmonary, alveolar and bronchial vessels. Plateletleukocyte interaction and NETosis are crucial players in acute lung injury (ALI), characterized by enhanced infiltration of neutrophils into the extravascular lung structures. Thus, platelets contribute to tissue damage and the severity of ALI via direct interaction with neutrophils and the release of proinflammatory mediators. TXA 2 release is a key mediator in Transfusion-Related Acute Lung Injury (TRALI) pathology. Recently, it has been demonstrated that aspirin intake can prevent the severity of the disease (50). On the other hand, aspirin itself can generate a chronic asthmatic disease called exacerbated respiratory disease (AERD) due to upregulation of cysteinyl leukotrienes, which leads to lung inflammation (51).

In addition, different responses in platelet function caused by antithrombotic drugs, such as clopidogrel, can be influenced by various factors such as genetic factors and demographic and clinical variables, including age $(52,53)$, diabetes mellitus, obesity, smoking (54), acute coronary syndromes, poor left ventricular function (55), and renal failure. As shown in Table 1, but not yet demonstrated in RCT trials, it is reasonable to consider that the action of distinctive receptors antagonists could interfere in different stages of platelet activation and result in a mixed quality of inhibition.

\section{Platelets in cancer and the co-existence of ITP}

Platelets can support tumour cell survival in circulation after they detach from the primary site and intravasate into blood vessels (55). Thrombocytosis has been associated with a poor prognosis in various types of cancers (57) and the roles of platelets in primary tumour growth and angiogenesis have been considerably discussed. In metastatic disease, the level of VEGF (vascular endothelial growth factor) is augmented and promotes blood vessel formation as an important step in tumour development. Platelet-tumour cell interactions and their contact with 


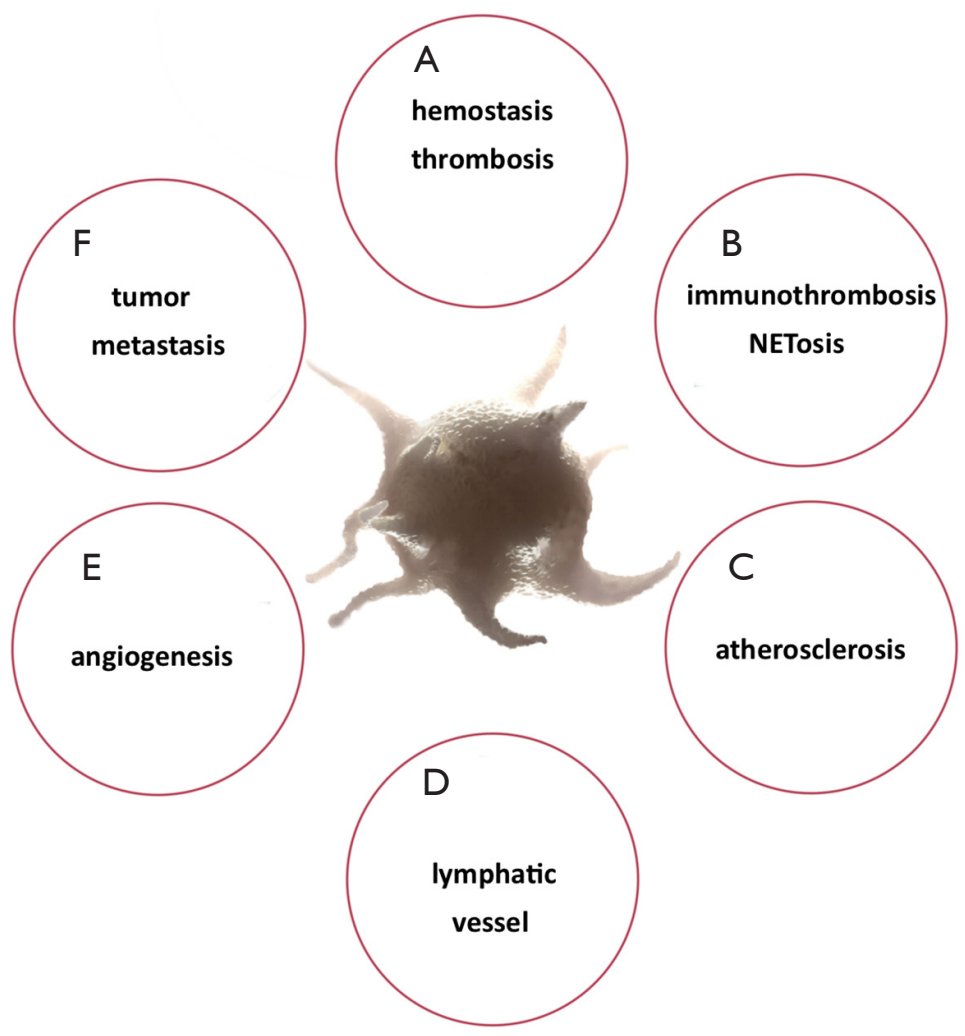

Figure 4 Platelets' versatility in health and diseases. (A) Platelets are crucial to haemostasis and thrombosis, in which state excessive platelet activation and aggregation can induce vessel occlusion which may culminate in stroke, venous thromboembolism and coronary syndromes. Thrombocytopenia or inherent or acquired platelet dysfunction may lead to bleeding disorders; (B) activated platelets bind to leukocytes and lymphocytes in blood and mediate recruitment to sites of injury and infection. Platelets are also involved in the NETosis process. NETs consist of a framework of filamentous DNA (chromatin) adorned with histone proteins, such as a web and several antibacterial components (e.g., neutrophil elastase and cathepsin G) that are expelled from activated neutrophils, which influence platelet function, particularly in the setting of infection and inflammation. The term "immuno-thrombosis" was recently coined to describe an innate immune response induced by the formation of thrombi in microvessels or other vascular beds (45); (C) platelets are involved in the arteriosclerosis process, through their activation, lipid metabolism and proinflammatory immune response; (D) platelets play crucial roles in the separation of the lymphatic system from the vasculature; (E) platelets potentiate or inhibit angiogenesis via the release of alpha-granules; (F) platelets promote tumour cell survival in circulation, tumour cell arrest and adhesion to the vascular wall, and tumour cell extravasation. NETs, neutrophil extracellular traps.

exposed subendothelial components involve key receptors such as GPIb, $\alpha \operatorname{IIb} \beta 3$, GPVI and exposed P-selectin (58). Observational case reports have shown an overlapping association of ITP (immune thrombocytopenia purpura) with breast cancer. The diagnosis of ITP might not prohibit a patient with breast cancer from receiving adjuvant chemotherapy since thrombocytopenia is a common complication of cancer, but it could be a contraindication for aggressive antineoplastic therapy until the aetiology of the low platelet count is determined and the clinical situation is successfully addressed (59).
ITP has been identified in patients with different types of cancer such as carcinoma of the lungs, ovaries, uterus, prostate, testes, bladder, and colon, and there have been 4 case reports of an association between breast cancer and ITP (60). Intriguingly, recently it has been reported that continuous intake of low-dose aspirin, excluding highdose aspirin, caused a decline in the long-term incidence of some cancer types (61). Aspirin reduces platelet activity (Figure 3), thus attenuating metastatic propagation of cancer cells. In mouse models, $\mathrm{P}_{2} \mathrm{Y}_{12}$ deficiency or its pharmacological inhibition significantly lowers 

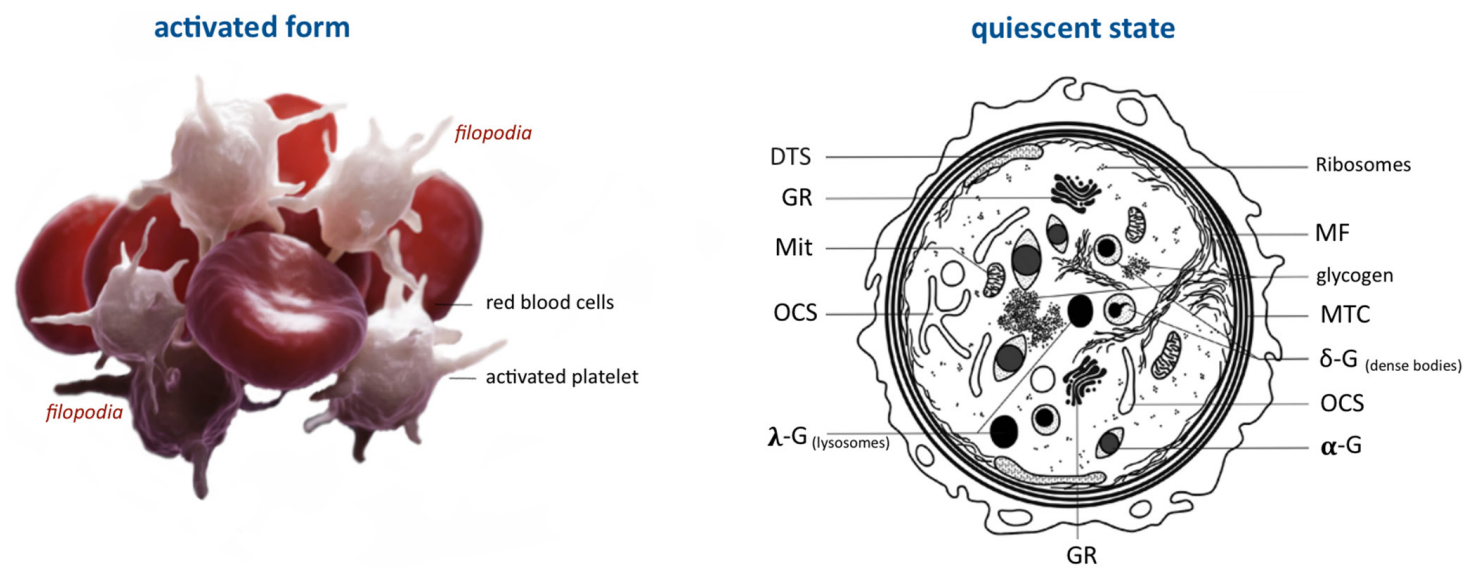

\begin{tabular}{|c|c|c|}
\hline & $\alpha$-Granules & Dense granules \\
\hline Diameter & $200-500 \mathrm{~nm}$ & $150 \mathrm{~nm}$ \\
\hline Number & $50-80$ per platelet & $3-8$ per platelet \\
\hline Percentage of cell volume & 10 & $\sim 1$ \\
\hline Contents & $\begin{array}{l}\text { Integral membrane proteins (e.g., P-selectin, } \\
\alpha \text { llb } \beta 3, \text { GPIb } \alpha \text { ) } \\
\text { Coagulants/anticoagulants and fibrinolytic } \\
\text { proteins (e.g., factor V, factor IX, } \\
\text { plasminogen) } \\
\text { Adhesion proteins (e.g., fibrinogen, VWF) } \\
\text { Chemokines [e.g., CXCL4 (PF4), CXCL12 } \\
\text { (SDF-1 } \alpha \text { )] } \\
\text { Growth factors (e.g., EGF, IGF) } \\
\text { Angiogenic factors/inhibitors (e.g., VEGF, } \\
\text { PDGF, angiostatins) }\end{array}$ & $\begin{array}{l}\text { Cations (e.g., } \mathrm{Ca}^{2+}, \mathrm{Mg}^{2+} \text { ) } \\
\text { Polyphosphates } \\
\text { Bioactive amines (e.g., } \\
\text { serotonin, histamine) } \\
\text { Nucleotides (e.g., ADP, ATP) }\end{array}$ \\
\hline
\end{tabular}

Figure 5 Schematic ultrastructure of platelets and granule types. The platelet is a 2-3 $\mu \mathrm{m}$ discoid cell with a remarkable amount of dynamic organelles that lacks a nucleus but contains $\alpha$-granules, dense granule and lysosomes. Platelet thickness is less than $1 \mu \mathrm{m}$. Platelets also contain Mit. Tunnel invaginations of the plasma membrane form a complex membrane network, termed the OCS, which courses throughout the platelet interior. Platelet granule secretion is thought to occur through the fusion of granules with either the plasma membrane or the OCS. The figure shows the content of the granules in a schematic view. PLTs become activated and change their shape dramatically after vessels are injured, acquiring an amoeboid form due to the rearrangement of organelles, triggered by microtubules (MTC) and microfilaments. During PLT activation, the MTC is primarily contracted and then fragmented and dislocated to the newly formed filopodia, here well-integrated with red blood cells (coloured scanning electron micrograph). The ultrastructure of PLTs, as well as the release reaction of granule contents during PLT activation, has been described in an excellent review by Rendu et al. (47). Alfa-Granules contains a variety of membrane proteins and soluble cargo that give them a distinct appearance. Proteomic analyses indicate that these granules contain hundreds of different types of proteins, including neuroactive peptides, tachykinins and enkephalins. Importantly, these can include coagulants and anticoagulants, angiogenic and antiangiogenic factors, proteases and proteases inhibitors, and pro-inflammatory and anti-inflammatory mediators. $\delta$-Gs are so electron dense that they can be detected using whole mount electron microscopy in the absence of staining. $\delta$-Gs play a critical role in haemostasis and thrombosis, releasing factors such as ADP and epinephrine that act in an autocrine and paracrine manner to stimulate platelets at sites of vascular injury. $\delta-\mathrm{G}$ also contain factors that are vasoconstrictive, such as serotonin, as well as high concentrations of cations, polyphosphates, adenine nucleotides, and bioactive amines, such as serotonin and histamine. During exocytosis PLT granules fuse with the OSC and their contents are released into the OSC and diffuse out into the extracellular environment. The cytoplasm contains $\alpha-$, $\delta$-, and $\lambda$-granules and the DTS which derives from the endoplasmic reticulum of MKs. Ribosomes and polyribosomes, as well as few Mit, are also present as equipment remaining from the MKs. PLTs also often contain a high number of accumulated glycogen (Gly) granules. Parts of the DTS line the marginal cytoskeletal ring. Resting discoid PLTs contain a peripheral stabilizing microtubular ring (MTC) built up of $\beta$-tubulin, which stabilizes platelets. The ring represents a circumferential MTC consisting of one microtubule wound 8-12 times stabilizing the PLT shape (drawing quiescent PLT in black). During PLT activation, the MTC is primary contracted and then fragmented and dislocated to the newly formed filopodia (colour cayenne). OCS, open canalicular system; MTC, MicroTubular Canalicular System; PLT, platelets; $\delta$-G, dense Granules; MK, megakaryocyte; Mit, mitochondria; DTS, dense tubular system. 


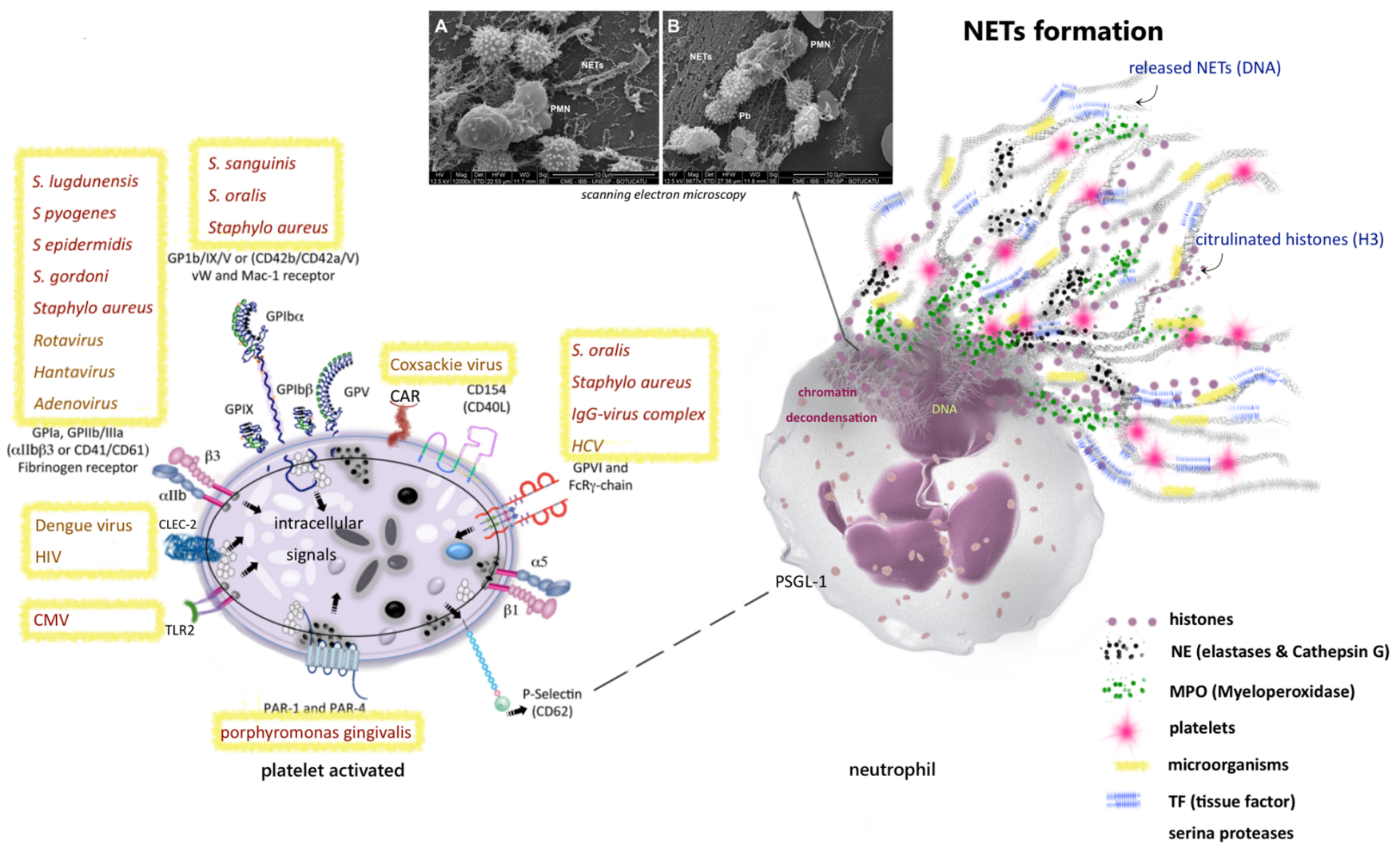

Figure 6 NETosis and the importance of platelet receptors in identifying microorganisms. Beyond the thrombotic effects of platelets, recently a cross-link interaction between neutrophils and platelets was described using the "NETosis system", characterized by the term Immuno-thrombosis. This scheme depicts activated platelets interacting with neutrophils involving P-Selectin and GPIb $\alpha / \alpha M \beta 2$ (not shown). Platelet receptors are also targets of microorganisms (viruses, fungi and bacteria, all represented in yellow boxes with yellow marks among the extracellular DNA strands). In addition, some microorganism-derived factors (e.g., bacterial lipopolysaccharide) interact either directly or indirectly via ligands, promoting neutrophil engagement and generation of NETs. Evidence has emerged that platelets and their activation state can also modulate innate and adaptive immune responses and low platelet counts (thrombocytopenia) have been identified as a surrogate marker for poor prognosis in septic patients and other diseases. From the evolutionary perspective, the cellular mediators of haemostasis and immune defences have not always been separated, such as the unique cells of the Limulus haemolymph (amoebocytes). In highly developed species, haemostasis and immune responses have been divided and leukocytes resume the immune response functions, while platelets maintain haemostasis. At the top inside of the figure, there is a real image collected by scanning electron microscopy showing that neutrophils (PMN cultures) when challenged with Pbs are able to release NETs to the extracellular environment (48). CMV, cytomegalovirus; GP, glycoprotein; HIV, human immunodeficiency virus; HCV, hepatitis virus; CAR, the coxsackie-adenovirus specific receptor expressed by platelets; CLEC-2, C-type lectin domain family 2; PAR-1/4, proteinase activated receptors; NETs, neutrophil extracellular traps; P. brasiliensis $(\mathrm{Pb})$.

metastases (62) and hepatocellular carcinoma (63). However, to raise more controversy, an RCT trial revealed that aspirin and clopidogrel had no effect on the number of circulating tumour cells in metastatic breast cancer (64). The following question has not yet been answered: can the administration of antithrombotic agents have benefits for primary and secondary cancer prevention?

\section{Neutrophil extracellular traps (NETs) tangled with platelets}

Neutrophils initiate host defences with phagocytosis, degranulation, cytokine production, oxidative bursts and, most recently described, NET construction (65). Neutrophils are the most populous innate immune cells 
Table 1 Different steps of platelet Activation described by Canizares et al. (56)

\begin{tabular}{lll}
\hline Structure & Activation level & Morphologic changes \\
\hline Discoid form & Quiescent form & Unaltered \\
$\begin{array}{l}\text { Pseudo tubular form } \\
\text { Membranous form }\end{array}$ & MTC disappears; OCS increases \\
Saccular stage & Slight activation & - \\
Pseudopodial stage & Moderate activation & Amoeboid PLT shape; saccular-tubular reorganizations; \\
& & an onset of degranulation \\
Hyaline stage & Strong activation & Degranulation completed; projections containing \\
\hline
\end{tabular}

MTC, MicroTubular Canalicular System; OCS, open canalicular system; PLT, platelets.

found in circulation, playing an indispensable role in surveying the body for signs of infection and inflammation. NETs were initially described as an antimicrobial mechanism of neutrophils; however, evidence suggests their involvement in a number of pathological conditions, such as deep vein thrombosis (DVT), autoimmune diseases (ANCAAssociated vasculitis, Systemic Lupus Erythematosus, and Psoriasis), diabetes, ALI, acute respiratory distress syndrome, cystic fibrosis, asthma, and cancer (66). Activated neutrophils undergo dramatic morphological changes in order to release NETs $(48,67,68)$ (Figure 6). The nuclear and granular membranes disintegrate and elastase enters into the nucleus, followed by hypercitrullination of histones, chromatin decondensation into the cytoplasm, rupture of the plasma membrane, and extrusion of nuclear material from the cell into the extracellular space. NETs have been shown to promote activation of select coagulation factors and platelets, thereby exacerbating several disease states including thrombosis (69). The term "immunothrombosis" was recently coined (70) to describe an innate immune response induced by the formation of thrombi in microvessels or other vascular beds. This response entangles immune cells and prothrombotic proteins including thrombin, active factors VIIa and Xa and tissue factor, to generate intravascular clots as a framework that accelerates the recognition, containment and destruction of pathogens. Thus, platelets have played an important function in this pathogen recognition mechanism (Figure 6).

\section{Role of platelets in infections}

When damage to the vascular system occurs, the host response is immediate, involving endothelial cells, platelets and neutrophils. Platelets rapidly adhere at the site of injury, to limit blood loss and initiate wound repair. Several receptors involved in platelet aggregation and adhesion also either engage bacteria or bacterial proteins directly or indirectly by binding to bridge molecules such as fibrinogen or vWf. Recently, Trugilho et al. (71) demonstrated that dengue virus is able to induce not only platelet activation and an inflammatory response but also antigen processing and presentation, proteasome activity, and the expression of histones, shedding light on new mechanisms of plateletmediated immune and inflammatory responses.

In Figure 6, an activated platelet is shown with specific proteins secreted by strains of gram-positive bacteria (e.g., Staphylococcus) and many viruses. In addition, the receptor FcRIIa has a critical role in the pathogenesis of heparin-induced thrombocytopenia (HIT), a potentially fatal disease where platelets are bound by temporary antigenic complexes of heparin, platelet factor $4(\mathrm{PF} 4)$ and $\mathrm{IgG}$ as an adverse event to exposure to large amounts of heparin. When administered in some patients, heparin can produce elevated amounts of heparin/ PF4-dependent autoantibodies, (HIT-autoantibodies) $\mathrm{PF} 4$, a tetrameric member of the $\mathrm{C}-\mathrm{X}-\mathrm{C}$ subfamily of chemokines, and form complexes with heparin-bound HIT antibodies. Furthermore, when bound to the platelet surface through the Fc portion, these complexes connect to immunoglobulins (IgG) and induce further FcRIIadependent platelet activation, leading to aggregation, thrombocytopenia and the onset of widespread thrombosis and inflammation. Concurrently, the FcRIIa receptor can be expressed on injured endothelium (72). Recent studies have shown that bacterial DNA may substitute for heparin to form similar antigenic complexes with comparable 
characteristics of platelet aggregation, thrombocytopenia and thrombosis $(73,74)$. Recently, it was identified that viruses such as Zika virus (ZIKV) can be transmitted by means of platelet transfusion, especially in persons with limited antibody response due to immunosuppressive therapy (75). Therefore, platelet interaction with different types of viruses has a great impact on platelet activation and platelet-mediated modulation of immunity (76). Platelets interact with other cells and microorganisms favouring immune-thrombosis in order to contain the spread of infections but can also promote transmission of infectious diseases when improperly transfused, leading to poor outcomes, especially in cancer patients. Intriguingly, the presence of thrombocytopenia may favour infections in the host in many ways and microorganism infections can trigger this phenomenon by different mechanisms (e.g., decreasing production or increasing destruction) linking their receptors directly or indirectly to other receptors, such as the Ashwell receptor that mitigates the lethal coagulopathy of sepsis by eliminating platelets desialylated by the bacterium's neuraminidase (e.g., Streptococcus pneumoniae) (77).

\section{Conclusions}

Future studies are coming and will open additional perspectives in order to enlighten our understanding from bench-to-bedside regarding these "discoid corpuscles", as elegantly described by Bizzozero and Osler in 1874. We are coping with an overflow of information and knowledge this century. Therefore, we have to develop a better approach to medical practice through optimized decision-making, emphasizing the correct use of evidence gathered from well-designed and well-conducted research, always keeping in mind values in healthcare. However, all of these tools and principles are not sufficient. It is necessary to integrate the best research evidence with clinical expertise and patient values, as defined by Sackett et al. (78). We have been learning about platelets since Bizzozero. In this endeavour, we have acquired skills to progress. Science is fed by curiosity and controversy, just as each platelet receptor connects with the appropriate ligand, regardless of the effects produced.

Finding the best way to treat and care for our patients extends beyond new discoveries and advancements and includes discoveries related to antithrombotic and anticoagulant agents in the management of cardiovascular diseases, cancer-related thrombotic diseases and even diabetes (79). Our greatest challenge is abandoning rooted habits that have the power to imprison hands and minds and block progress.

The scientific path should not be askew similar to Limulus (Limulus means crooked in Greek) or savage similar to Polyphemus (a man-eating giant, son of Poseidon in Greek mythology) as both can lead to devastating and undesirable results, a product of careless medicine.

\section{Acknowledgements}

None.

\section{Footnote}

Conflicts of Interest: The authors have no conflicts of interest to declare.

\section{References}

1. Coller BS. A brief history of ideas about platelets in health and disease. In: Michelson AD, editor. Platelets. Third edition. San Diego: Elsevier/Academic Press, 2013:19-53.

2. Ribatti D, Crivellato E. Giulio Bizzozero and the discovery of platelets. Leuk Res 2007;31:1339-41.

3. Robb-Smith AH. Why the platelets were discovered. Br J Haematol 1967;13:618-37.

4. Mazzarello P, Calligaro AL, Calligaro A. Giulio Bizzozero: a pioneer of cell biology. Nat Rev Mol Cell Biol 2001;2:776-81.

5. Levin J. The evolution of mammalian platelets. In: Michelson AD, editor. Platelets. Third edition. San Diego: Elsevier/Academic Press, 2013:3-25.

6. Bayer AS, Sullam PM, Ramos M, et al. Staphylococcus aureus induces platelet aggregation via a fibrinogendependent mechanism which is independent of principal platelet glycoprotein IIb/IIIa fibrinogen-binding domains. Infect Immun 1995;63:3634-41.

7. Harrington M. Blue-bloods of the sea. Lab Anim (NY) 2015;44:83.

8. Göpel T, Wirkner CS. An "ancient" complexity? Evolutionary morphology of the circulatory system in Xiphosura. Zoology 2015;118:221-38.

9. Maule J, Morris H, Monaco L, et al. Rapid Biochemical Analysis on the International Space Station (ISS): Preparing for Human Exploration of the Moon and Mars. NASA Technical Reports Server 2008. Available online: https://ntrs.nasa.gov/search.jsp?R=20090008540

10. Fennrich S, Hennig U, Toliashvili L, et al. More than 
70 years of pyrogen detection: Current state and future perspectives. Altern Lab Anim 2016;44:239-53.

11. 2016 top markets report medical devices 2016. Available online: http://www.trade.gov/topmarkets/pdf/Medical_ Devices_Executive_Summary.pdf

12. Cartwright-Taylor L, Lee J, Hsu CC. Population structure and breeding pattern of the mangrove horseshoe crab Carcinoscorpius rotundicauda in Singapore. Aquatic Biology 2009;8:61-9.

13. Ruggeri ZM. Platelets in atherothrombosis. Nat Med 2002;8:1227-34.

14. Lindemann S, Krämer B, Seizer P, et al. Platelets, inflammation and atherosclerosis. J Thromb Haemost 2007;5 Suppl 1:203-11.

15. Li C, Li J, Li Y, et al. Crosstalk between Platelets and the Immune System: Old Systems with New Discoveries. Adv Hematol 2012;2012:384685.

16. Hess PR, Rawnsley DR, Jakus Z, et al. Platelets mediate lymphovenous hemostasis to maintain blood-lymphatic separation throughout life. J Clin Invest 2014;124:273-84.

17. Wang Y, Hayes V, Jarocha D, et al. Comparative analysis of human ex vivo-generated platelets vs megakaryocytegenerated platelets in mice: a cautionary tale. Blood 2015;125:3627-36.

18. Aschoff L. Ueber capillare embolie von riesenkernhaltigen zellen. Arch Pathol Anat Physiol 1893;134:11À4.

19. Italiano JE, Hartwig JH. Megakaryocyte development and platelet formation. In: Michelson AD, editor. Platelets. 3rd edition. Amsterdam: Elsevier/Academic Press, 2013:27-49.

20. Nishimura S, Nagasaki M, Kunishima S, et al. IL-1alpha induces thrombopoiesis through megakaryocyte rupture in response to acute platelet needs. J Cell Biol 2015;209:453-66.

21. Kile BT. The role of apoptosis in megakaryocytes and platelets. Br J Haematol 2014;165:217-26.

22. Mason KD, Carpinelli MR, Fletcher JI, et al. Programmed anuclear cell death delimits platelet life span. Cell 2007;128:1173-86.

23. Grozovsky R, Giannini S, Falet H, et al. Regulating billions of blood platelets: glycans and beyond. Blood 2015;126:1877-84.

24. Hoffman M, Monroe DM 3rd. A cell-based model of hemostasis. Thromb Haemost 2001;85:958-65.

25. Hoffman M, Monroe DM. Coagulation 2006: a modern view of hemostasis. Hematol Oncol Clin North Am 2007;21:1-11.

26. Wang Y, Reheman A, Spring CM, et al. Plasma fibronectin supports hemostasis and regulates thrombosis. J Clin Invest 2014;124:4281-93.
27. Wang Y, Ni H. Fibronectin maintains the balance between hemostasis and thrombosis. Cell Mol Life Sci 2016;73:3265-77.

28. Wang Y, Gallant RC, Ni H. Extracellular matrix proteins in the regulation of thrombus formation. Curr Opin Hematol 2016;23:280-7.

29. Lea Beaulieu JF. Inhibition of platelet function by the endothelium. In: Michelson AD, editor. Platelets. 3rd edition. San Diego: Elsevier/Academic Press, 2013:313-42.

30. Clemetson KJ, Clemetson JM. Platelet receptors. In: Michelson AD, editor. Platelets. 3rd edition. San Diego: Elsevier/Academic Press, 2013:169-94.

31. Mackman N. Triggers, targets and treatments for thrombosis. Nature 2008;451:914-8.

32. Franchi F, Angiolillo DJ. Novel antiplatelet agents in acute coronary syndrome. Nat Rev Cardiol 2015;12:30-47.

33. Franchini M, Manzato F, Salvagno GL, et al. Potential role of recombinant activated factor VII for the treatment of severe bleeding associated with disseminated intravascular coagulation: a systematic review. Blood Coagul Fibrinolysis 2007;18:589-93.

34. Gachet C. Antiplatelet drugs: which targets for which treatments? J Thromb Haemost 2015;13 Suppl 1:S313-22.

35. Metharom P, Berndt MC, Baker RI, et al. Current state and novel approaches of antiplatelet therapy. Arterioscler Thromb Vasc Biol 2015;35:1327-38.

36. Writing Group Members, Mozaffarian D, Benjamin EJ, et al. Heart Disease and Stroke Statistics-2016 Update: A Report From the American Heart Association. Circulation 2016;133:e38-360.

37. Cavender MA, Scirica BM, Bonaca MP, et al. Vorapaxar in patients with diabetes mellitus and previous myocardial infarction: findings from the thrombin receptor antagonist in secondary prevention of atherothrombotic ischemic events-TIMI 50 trial. Circulation 2015;131:1047-53.

38. Frampton JE. Vorapaxar: a review of its use in the longterm secondary prevention of atherothrombotic events. Drugs 2015;75:797-808.

39. Moon JY, Franchi F, Rollini F, et al. Role for Thrombin Receptor Antagonism With Vorapaxar in Secondary Prevention of Atherothrombotic Events: From Bench to Bedside.J Cardiovasc Pharmacol Ther 2017. [Epub ahead of print].

40. Khunger N. Accelerated Wound Healing: Harnessing the Power of Platelets, Biomaterials, Stem Cells and Gene Therapy. J Cutan Aesthet Surg 2017;10:1-2.

41. Michelson AD. Platelets. 3rd edition. UK: Elsevier and Academic Press, 2013.

42. Nurden AT, Nurden P, Sanchez M, et al. Platelets and 
wound healing. Front Biosci 2008;13:3532-48.

43. Sudoyo AW, Rachman A, Harimurti K. Angiogenesis, inflammation, platelets count, and metastatic status as a predictor for thrombosis risk in nasopharyngeal carcinoma patients. Acta Med Indones 2015;47:11-5.

44. Trier DA, Gank KD, Kupferwasser D, et al. Platelet antistaphylococcal responses occur through P2X1 and $\mathrm{P} 2 \mathrm{Y} 12$ receptor-induced activation and kinocidin release. Infect Immun 2008;76:5706-13.

45. Andrews RK, Arthur JF, Gardiner E. Neutrophil extracellular traps (NETs) and the role of platelets in infection. Thromb Haemost 2014;112:659-65.

46. White J. Platelet structure. In: Michelson AD, editor. Platelets. San Diego: Elsevier/Academic Press, 2013:117-44.

47. Rendu F, Brohard-Bohn B. The platelet release reaction: granules' constituents, secretion and functions. Platelets 2001;12:261-73.

48. Brinkmann V, Goosmann C, Kuhn LI, et al. Automatic quantification of in vitro NET formation. Front Immunol 2013;3:413.

49. Schrottmaier WC, Kral JB, Badrnya S, et al. Aspirin and P2Y12 Inhibitors in platelet-mediated activation of neutrophils and monocytes. Thromb Haemost 2015;114:478-89.

50. Caudrillier A, Looney MR. Platelet-neutrophil interactions as a target for prevention and treatment of transfusionrelated acute lung injury. Curr Pharm Des 2012;18:3260-6.

51. Laidlaw TM, Kidder MS, Bhattacharyya N, et al. Cysteinyl leukotriene overproduction in aspirinexacerbated respiratory disease is driven by plateletadherent leukocytes. Blood 2012;119:3790-8.

52. Trenk D, Hochholzer W, Fromm MF, et al. Cytochrome P450 2C19 681G>A polymorphism and high onclopidogrel platelet reactivity associated with adverse 1-year clinical outcome of elective percutaneous coronary intervention with drug-eluting or bare-metal stents. J Am Coll Cardiol 2008;51:1925-34.

53. Simon T, Verstuyft C, Mary-Krause M, et al. Genetic determinants of response to clopidogrel and cardiovascular events. N Engl J Med 2009;360:363-75.

54. Bliden KP, Dichiara J, Lawal L, et al. The association of cigarette smoking with enhanced platelet inhibition by clopidogrel. J Am Coll Cardiol 2008;52:531-3.

55. Labelle M, Begum S, Hynes RO. Platelets guide the formation of early metastatic niches. Proc Natl Acad Sci USA 2014;111:E3053-61.

56. Cañizares C, Vivar N, Tenesaca S. Analysis of the ultrastructure of the platelets during the process of aggregation, with emphasis in the cytoskeleton and membranous changes. Microsc Electron Biol Celular 1988;12:1-15.

57. Gay LJ, Felding-Habermann B. Contribution of platelets to tumour metastasis. Nat Rev Cancer 2011;11:123-34.

58. Kim YJ, Borsig L, Varki NM, et al. P-selectin deficiency attenuates tumor growth and metastasis. Proc Natl Acad Sci USA 1998;95:9325-30.

59. Peffault de Latour R, Des Guetz G, Laurence V, et al. Breast cancer associated with idiopathic thrombocytopenic purpura: a single center series of 10 cases. Am J Clin Oncol 2004;27:333-6.

60. Wahid FS, Fun LC, Keng CS, et al. Breast carcinoma presenting as immune thrombocytopenic purpura. Int J Hematol 2001;73:399-400.

61. Rothwell PM, Wilson M, Price JF, et al. Effect of daily aspirin on risk of cancer metastasis: a study of incident cancers during randomised controlled trials. Lancet 2012;379:1591-601.

62. Wang Y, Sun Y, Li D, et al. Platelet P2Y12 is involved in murine pulmonary metastasis. PLoS One 2013;8:e80780.

63. Sitia G, Iannacone M, Guidotti LG. Anti-platelet therapy in the prevention of hepatitis B virus-associated hepatocellular carcinoma. J Hepatol 2013;59:1135-8.

64. Roop RP, Naughton J, Poznak VC, et al. A randomized phase ii trial investigating the effect of platelet function inhibition on circulating tumor cells in patients with metastatic breast cancer. Clin Breast Cancer 2013;13:409-15.

65. Brinkmann V, Reichard U, Goosmann C, et al. Neutrophil extracellular traps kill bacteria. Science 2004;303:1532-5.

66. Mitsios A, Arampatzioglou A, Arelaki S, et al. NETopathies? Unraveling the Dark Side of Old Diseases through Neutrophils. Front Immunol 2017;7:678.

67. Wang Y, Li M, Stadler S, et al. Histone hypercitrullination mediates chromatin decondensation and neutrophil extracellular trap formation. J Cell Biol 2009;184:205-13.

68. Della Coletta AM, Bachiega TF, de Quaglia e Silva JC, et al. Neutrophil Extracellular Traps Identification in Tegumentary Lesions of Patients with Paracoccidioidomycosis and Different Patterns of NETs Generation In Vitro. PLoS Negl Trop Dis 2015;9:e004037.

69. Healy LD, Puy C, Fernandez JA, et al. Activated protein $\mathrm{C}$ inhibits neutrophil extracellular trap formation in vitro and activation in vivo. J Biol Chem 2017;292:8616-29.

70. Engelmann B, Massberg S. Thrombosis as an intravascular effector of innate immunity. Nat Rev Immunol $2013 ; 13: 34-45$. 
71. Trugilho MRO, Hottz ED, Brunoro GVF, et al. Platelet proteome reveals novel pathways of platelet activation and platelet-mediated immunoregulation in dengue. PLoS Pathog 2017;13:e1006385.

72. Bakchoul T, Greinacher A, Warkentin TE. Heparininduced thrombocytopenia in 2017 and beyond. Thromb Haemost 2016;116:781-2.

73. Krauel K, Potschke C, Weber C, et al. Platelet factor 4 binds to bacteria, [corrected] inducing antibodies crossreacting with the major antigen in heparin-induced thrombocytopenia. Blood 2011;117:1370-8.

74. Krauel K, Weber C, Brandt S, et al. Platelet factor 4 binding to lipid A of Gram-negative bacteria exposes PF4/ heparin-like epitopes. Blood 2012;120:3345-52.

Cite this article as: Costa-Filho RC, Bozza FA. Platelets: an outlook from biology through evidence-based achievements in critical care. Ann Transl Med 2017;5(22):449. doi: 10.21037/ atm.2017.11.04
75. Motta IJ, Spencer BR, Cordeiro da Silva SG, et al. Evidence for Transmission of Zika Virus by Platelet Transfusion. N Engl J Med 2016;375:1101-3.

76. Assinger A. Platelets and infection - an emerging role of platelets in viral infection. Front Immunol 2014;5:649.

77. Grewal PK, Uchiyama S, Ditto D, et al. The Ashwell receptor mitigates the lethal coagulopathy of sepsis. Nat Med 2008;14:648-55.

78. Sackett DL. Evidence-based medicine and treatment choices. Lancet 1997;349:570; author reply 572-3.

79. Xu XR, Carrim N, Neves MA, et al. Platelets and platelet adhesion molecules: novel mechanisms of thrombosis and anti-thrombotic therapies. Thromb J 2016;14:29. 